\title{
Early management of sepsis
}

elSSN: $2383-4625$

\section{Jean-Louis Vincent ${ }^{1}$, Adriano José Pereira ${ }^{2}$, James Gleeson' ${ }^{1}$, Daniel De Backer ${ }^{1}$}

'Department of Intensive Care, Erasme Hospital, Université Libre de Bruxelles, Brussels, Belgium

${ }^{2}$ Department of Intensive Care Medicine, Hospital Israelita Albert Einstein, São Paulo, Brazil

Increased awareness of the signs and symptoms of sepsis and an emphasis on the importance of early treatment have helped to improve survival rates from this serious and frequent condition in recent years. With no specific, effective anti-sepsis therapies available, management focuses on early source control with adequate and appropriate antibiotics and removal of any source of infection, rapid resuscitation, hemodynamic stabilization and organ support. Use of dedicated teams to care for patients with sepsis can help optimize early management.

Keywords Sepsis; Survival; Resuscitation; Hemodynamics; Management teams

Capsule
Wummat is already known
Sepsis affects a large proportion of the critically ill population and is associated
with considerable morbidity and mortality. No effective specific anti-sepsis
treatments are commercially available and management relies on administra-
tion of antibiotics, source control measures, effective resuscitation strategies,
and organ support.
What is new in the current study
Increased awareness of sepsis and the importance of early treatment has
helped to reduce mortality rates from sepsis in recent years. Use of protocols
may be helpful in hospitals where usual care is substandard or in units that are
less familiar with the latest recommendations for sepsis management. Use of
dedicated teams to care for patients with sepsis will help optimize early man-
agement.

Received: 22 June 2014

Revised: 15 July 2014

Accepted: 11 August 2014

Correspondence to: Jean-Louis Vincent Department of Intensive Care, Erasme Hospital, Route de Lennik 808, B-1070 Brussels, Belgium E-mail: jlvincen@ulb.ac.be

\section{How to cite this article:}

Vincent JL, Pereira AJ, Gleeson J, De Backer D. Early management of sepsis. Clin Exp Emerg Med 2014;1(1):3-7.

This is an Open Access article distributed under the terms of the Creative Commons Attribution Non-Commercial License (http:// creativecommons.org/licenses/by-nc/3.0/). 


\section{INTRODUCTION}

Sepsis is defined as a severe infection with some degree of associated organ dysfunction ${ }^{1}$ and affects a large proportion of the critically ill population. Survival rates for patients with sepsis have increased over the last decade, largely related to improvements in intensive care in general rather than to any specific advance in sepsis therapies. Nevertheless, mortality rates remain at least 20\% in patients with septic shock and, despite multiple studies and potential candidates over the years, no effective specific anti-sepsis treatments are commercially available. In recent years, it has become clear that perhaps the most important aspect of the management of patients with sepsis is early recognition so that administration of antibiotics, source control measures and effective resuscitation strategies can be started as soon as possible after onset. Early management with adequate antimicrobials and rapid resuscitation to restore and stabilize hemodynamic status has been shown to be associated with improved outcomes $^{3,4}$ so that every attempt should be made to diagnose sepsis as soon as possible. However, diagnosis of sepsis is not always easy, especially in critically ill patients with other conditions and signs and symptoms that can mimic severe infection. Most signs and variables that are known to be associated with sepsis (e.g., fever, tachycardia, and tachypnea) are not specific for sepsis, but their presence can alert the physician to a possible diagnosis and encourage further investigations. Other factors, including raised biomarker levels, e.g., C-reactive protein and/or procalcitonin, Xray images suggesting infection, presence of unexplained organ dysfunction, can all add up to increase the likelihood of a sepsis diagnosis.

Once a diagnosis is established, appropriate management is a priority and timing becomes crucial. The management of septic shock can be considered in four phases according to the salvage, optimization, stabilization, de-escalation (SOSD) mnemonic. ${ }^{5}$ In this review, we will concentrate on some key aspects of the early, salvage and optimization phases of sepsis management.

\section{EARLY SOURCE CONTROL AND ANTIMICROBIAL THERAPY}

Any approach to treatment of patients with sepsis will be ineffective if the underlying infectious cause is not eliminated. Once a diagnosis of sepsis is suspected, a thorough search for the likely source must be conducted, remembering the big 5 common culprits: lungs, abdomen, urine, wounds, and indwelling devices (especially catheters in hospitalized patients). Clinical signs and symptoms, appropriate microbiological cultures, and relevant im- aging techniques must be used to try and determine where the infection is sited. Eradication of the source then involves not only adequate antimicrobial agents but also removal of any focus of infection, including iatrogenic causes such as drains or intravascular catheters. Surgical intervention may be necessary in some cases, e.g., if the infection is the result of a perforated diverticulum or an intra-abdominal abscess not amenable to percutaneous drainage.

Adequate antibiotic medication must be started as soon as possible-the Surviving Sepsis Campaign (SSC) guidelines recommend (grade 1B) that antimicrobials be started within 1 hour of diagnosis in patients with septic shock. ${ }^{6}$ Antimicrobial treatment should not be delayed while waiting for cultures to be taken, although when possible to perform rapidly, relevant cultures (e.g., blood, urine, and sputum) should ideally be collected before antibiotics are started.

Importantly, although early antibiotic therapy is crucial to optimize chances of survival, ${ }^{4}$ it will only be effective if the chosen antimicrobials are appropriate to the infection in question, i.e., the infecting microorganism is sensitive to the antibiotic given. The importance of initial appropriate antibiotics has been reported in multiple studies, being associated with improved survival rates compared to initial inappropriate antibiotics. ${ }^{7.8}$ Culture results generally take several days to become available, so it is usually necessary to start broad-spectrum empiric therapy that will cover all likely organisms, based on likely source of infection, local microbiological flora and resistance patterns, recent antimicrobial therapy, and healthcare facility exposure status. Combination empirical antimicrobial treatment has been shown to be associated with better outcomes than single-agent therapy in observational studies, ${ }^{9,10}$ but randomized controlled trials are needed to confirm these findings. Once culture results are available, the choice of antibiotics should be re-evaluated and de-escalation of antimicrobial treatment to a narrower spectrum should be performed whenever possible. This approach will optimize treatment efficacy, limit toxicity, help prevent development of resistance and reduce costs. ${ }^{11}$ Nevertheless, de-escalation may not be possible in all patients and will be dependent on the initial choice of empiric antibiotics and the culture results when they become available. In as many as $30 \%$ of patients culture results will be negative ${ }^{12,13}$ and many physicians will then prefer to continue broad-spectrum coverage. One study in an intensive care unit (ICU) with good collaboration between intensivists and infectious disease specialists reported that de-escalation was possible in only $43 \%$ of cases; however in only 5\% was the chance to de-escalate missed, in all other cases there was a sound reason not to narrow the spectrum. ${ }^{14}$ 
Adequate antimicrobial therapy refers not only to the appropriateness in terms of spectrum of cover, but also to the sufficient dose, route and duration of therapy. Antibiotics are typically given intravenously for 7 to 10 days but longer courses may be indicated in patients with slow clinical response, an undrainable focus of infection, for some fungal and viral infections, or in patients with immunologic deficiencies, including neutropenia (SSC guidelines grade $2 \mathrm{C}$ ). ${ }^{6}$ The use of biomarkers, notably procalcitonin, to guide antimicrobial therapy has been associated with reduced antimicrobial exposure and shorter lengths of ICU stay ${ }^{15-17}$ but not with improved survival and some studies have even suggested that this approach could be harmful. ${ }^{18,19}$ Further studies are needed to evaluate how these biomarkers could be used to help clinical decision making.

The recommended doses for many of the antibiotics used in patients with sepsis are derived from non-critically ill patients or healthy volunteers. Yet, the pharmacodynamics and kinetics of many antimicrobials will be altered in critically ill patients such that general recommended doses may be inadequate. ${ }^{20}$ In addition, organ dysfunction, particularly renal, and therapies, such as hemodialysis/hemofiltration, can also influence antimicrobial distribution and clearance, necessitating dose adaptation. Several studies have now been published investigating these issues in patients with sepsis ${ }^{21,22}$ but until precise dose recommendations can be made in specific situations, doses should be adapted to the individual patient to ensure that coverage is adequate. Where available, daily monitoring of antimicrobial levels can help in the attainment of therapeutic concentrations. ${ }^{23-25}$

\section{HEMODYNAMIC STABILIZATION AND EARLY GOAL-DIRECTED THERAPY}

In 2001, Rivers et al. ${ }^{3}$ published the results of a single-center study showing that early goal-directed therapy (EGDT) guided by central-venous oxygen saturation $\left(\mathrm{ScvO}_{2}\right)$ monitoring was associated with reduced mortality rates in emergency department patients with severe sepsis or septic shock compared to standard management. In addition to predefined central venous pressure and arterial pressure levels, the EGDT strategy consisted of a 6hour protocol titrating intravenous fluids, inotropes and packed red-cell transfusion to maintain $\mathrm{ScvO}_{2}$ above $70 \%$. However, the single-center nature of the study, concerns about possible lack of adequate management in the control arm, and debate about the specific components included in the protocol led some to question its relevance to all patients with severe sepsis. Moreover, standard patient management has altered since 2001, for example in terms of tighter blood glucose targets, use of lower tidal volumes, and lower blood transfusion triggers. Several multicenter randomized controlled trials were therefore conceived to assess the importance of the different components and the EGDT concept in general. The results of the first of these studies have now been published. The Protocolized Care for Early Septic Shock (PRoCESS) study in 31 emergency departments across the USA compared two protocolized groups (a Rivers-like EGDT protocol group and a protocol-based standard therapy group who were managed without placement of a central venous catheter, administration of inotropes, or blood transfusions) with a usual treatment arm. ${ }^{26}$ The results showed that protocol-based resuscitation of emergency department patients with septic shock was not associated with improved outcomes compared to usual care. Importantly, all the patients in the three arms had septic shock diagnosed early and received antibiotics and other non-resuscitation aspects of care promptly; moreover, all three groups of patients received aggressive fluid resuscitation prior to randomization and the $\mathrm{ScvO}_{2}$ was already within targets in most patients at randomization. These results suggest that the most important aspects of care are early diagnosis and rapid effective fluid resuscitation and antimicrobial therapy.

When considering the early phase of shock treatment, the VIP (ventilation, infusion, and pump) mnemonic proposed by Weil and Shubin ${ }^{27}$ remains a useful guide. All patients with septic shock must be adequately oxygenated to correct hypoxemia. Endotracheal intubation and mechanical ventilation will be needed in severe cases; non-invasive ventilation is not recommended. Hypovolemia needs to be corrected rapidly. Which fluid should be used in sepsis has been debated at length, but the quantity is more important than the type. Current guidelines recommend use of crystalloids as the first choice fluid for resuscitation (grade 1B) and albumin is suggested when large amounts of crystalloids are required (grade $2 \mathrm{C}$ ). ${ }^{6}$ Hydroxyethyl starch solutions are no longer recommended in sepsis. The amount of fluid administered will depend on the phase of shock, ${ }_{1}^{5}$ but in the early, salvage (S) phase, fluid administration should be generous; the SSC guidelines recommend starting with a fixed amount of about $30 \mathrm{~mL} / \mathrm{kg} .{ }^{6}$ Vasoactive agents are also often required and are frequently started during fluid administration to help avoid prolonged hypotension, which can impair tissue perfusion. Norepinephrine is the vasopressor of choice (grade 1B) because dopamine has been associated with increased adverse effects in patients with shock and higher mortality rates than norepinephrine in patients with septic shock. ${ }^{28,29}$ Low doses of dobutamine (about $5 \mathrm{mcg} / \mathrm{kg} / \mathrm{min}$ ) are often added to norepinephrine to increase cardiac output by its positive inotropic effects. As suggested by the PRoCESS trial, invasive monitoring to guide therapy is not necessary in all patients with septic 
shock. Monitoring changes in blood lactate levels can provide a good indication of the effectiveness of the resuscitation. ${ }^{30}$

\section{THE SEPSIS TEAM}

Despite increased awareness of the importance of early diagnosis and rapid appropriate treatment of patients with severe sepsis, many patients still do not receive acceptable early management. One of the reasons for this shortfall is that patients with sepsis are highly complex, often with multiple comorbidities and rapidly changing hemodynamics. The management of such patients involves multiple elements, including insertion of intravenous/arterial lines and setting up of hemodynamic monitoring systems, blood sampling for cultures and laboratory testing, administration of antibiotics, fluid resuscitation, and administration of vasoactive agents for cardiovascular support, all of which need to be started as soon as possible. The only realistic way of being able to simultaneously perform all the necessary tests and start the appropriate treatments is for initial management and stabilization to be organized by a 'sepsis team. Similar to the trauma teams now widely established for the management of patients with severe trauma and crash teams for patients with in-hospital cardiorespiratory arrest, patients with severe sepsis can be better managed by a team that includes several doctors and nurses as a minimum, but also possibly an infectious diseases specialist, radiographers, phlebotomist, pharmacist, and surgeon, depending on local resources. In such teams, each member will have their own predefined role to insure that all the essential aspects of initial management are covered. One member of the team would be clearly identified as the leader to direct and coordinate the overall management process. The 'sepsis team' should be available 24/7. In our hospital, we have a dedicated 'shock room' rather than a mobile sepsis team, which is staffed permanently by a team of nurses and doctors trained in shock management, including septic shock, and is equipped with all the necessary monitoring devices, intravascular lines and phlebotomy equipment, a ventilator ready for use, and essential intravenous solutions and drugs. ${ }^{31}$

Increasing numbers of hospitals are now developing code sepsis teams and studies support the positive impact of this approach on patient outcomes. ${ }^{32,33}$

\section{CONCLUSION}

Increased awareness of sepsis and an emphasis on the importance of early treatment has helped to improve survival rates in recent years, but a continued focus on early source control with adequate antibiotics and rapid resuscitation and hemodynamic stabilization must be maintained if outcomes are to improve further. Use of protocols per se does not improve survival in patients with septic shock, but this approach may be of use in hospitals where usual care is substandard or in units that are less familiar with the latest recommendations for sepsis management. Use of dedicated teams to care for patients with sepsis will help optimize early management.

\section{CONFLICT OF INTEREST}

No potential conflict of interest relevant to this article was reported.

\section{REFERENCES}

1. Vincent JL, Opal SM, Marshall JC, Tracey KJ. Sepsis definitions: time for change. Lancet 2013;381:774-5.

2. Kaukonen KM, Bailey M, Suzuki S, Pilcher D, Bellomo R. Mortality related to severe sepsis and septic shock among critically ill patients in Australia and New Zealand, 2000-2012. JAMA 2014;311:1308-16.

3. Rivers E, Nguyen B, Havstad S, et al. Early goal-directed therapy in the treatment of severe sepsis and septic shock. N Engl J Med 2001;345:1368-77.

4. Gaieski DF, Mikkelsen ME, Band RA, et al. Impact of time to antibiotics on survival in patients with severe sepsis or septic shock in whom early goal-directed therapy was initiated in the emergency department. Crit Care Med 2010;38:1045-53.

5. Vincent JL, De Backer D. Circulatory shock. N Engl J Med 2013; 369:1726-34.

6. Dellinger RP, Levy MM, Rhodes A, et al. Surviving Sepsis Campaign: international guidelines for management of severe sepsis and septic shock, 2012. Intensive Care Med 2013;39:165228.

7. Zahar JR, Timsit JF, Garrouste-Orgeas $M$, et al. Outcomes in severe sepsis and patients with septic shock: pathogen species and infection sites are not associated with mortality. Crit Care Med 2011;39:1886-95.

8. Kumar A, Ellis $P$, Arabi $Y$, et al. Initiation of inappropriate antimicrobial therapy results in a fivefold reduction of survival in human septic shock. Chest 2009;136:1237-48.

9. Kumar A, Zarychanski R, Light B, et al. Early combination antibiotic therapy yields improved survival compared with monotherapy in septic shock: a propensity-matched analysis. Crit Care Med 2010;38:1773-85.

10. Micek ST, Welch EC, Khan J, et al. Empiric combination antibiotic therapy is associated with improved outcome against sepsis due to Gram-negative bacteria: a retrospective analysis. 
Antimicrob Agents Chemother 2010;54:1742-8.

11. Masterton RG. Antibiotic de-escalation. Crit Care Clin 2011; 27:149-62.

12. Vincent JL, Sakr Y, Sprung CL, et al. Sepsis in European intensive care units: results of the SOAP study. Crit Care Med 2006;34:344-53.

13. Vincent JL, Rello J, Marshall J, et al. International study of the prevalence and outcomes of infection in intensive care units. JAMA 2009;302:2323-9.

14. Heenen $S$, Jacobs $F$, Vincent JL. Antibiotic strategies in severe nosocomial sepsis: why do we not de-escalate more often? Crit Care Med 2012;40:1404-9.

15. Nobre V, Harbarth S, Graf JD, Rohner P, Pugin J. Use of procalcitonin to shorten antibiotic treatment duration in septic patients: a randomized trial. Am J Respir Crit Care Med 2008; 177:498-505.

16. Bouadma L, Luyt CE, Tubach $F$, et al. Use of procalcitonin to reduce patients' exposure to antibiotics in intensive care units (PRORATA trial): a multicentre randomised controlled trial. Lancet 2010;375:463-74.

17. Prkno A, Wacker C, Brunkhorst FM, Schlattmann P. Procalcitonin-guided therapy in intensive care unit patients with severe sepsis and septic shock: a systematic review and meta-analysis. Crit Care 2013;17:R291.

18. Jensen JU, Hein L, Lundgren B, et al. Procalcitonin-guided interventions against infections to increase early appropriate antibiotics and improve survival in the intensive care unit: a randomized trial. Crit Care Med 2011;39:2048-58.

19. Heyland DK, Johnson AP, Reynolds SC, Muscedere J. Procalcitonin for reduced antibiotic exposure in the critical care setting: a systematic review and an economic evaluation. Crit Care Med 2011;39:1792-9.

20. McKenzie C. Antibiotic dosing in critical illness. J Antimicrob Chemother 2011;66 Suppl 2:ii25-31.

21. Seyler $L$, Cotton F, Taccone FS, et al. Recommended $\beta$-lactam regimens are inadequate in septic patients treated with continuous renal replacement therapy. Crit Care 2011;15:R137.
22. Taccone FS, Laterre PF, Dugernier T, et al. Insufficient $\beta$-lactam concentrations in the early phase of severe sepsis and septic shock. Crit Care 2010;14:R126.

23. Casu $G S$, Hites $M$, Jacobs $F$, et al. Can changes in renal function predict variations in $\beta$-lactam concentrations in septic patients? Int J Antimicrob Agents 2013;42:422-8.

24. Delattre IK, Musuamba F, Verbeeck RK, et al. Empirical models for dosage optimization of four beta-lactams in critically ill septic patients based on therapeutic drug monitoring of amikacin. Clin Biochem 2010;43:589-98.

25. Hites $M$, Taccone FS, Wolff $F$, et al. Case-control study of drug monitoring of $\beta$-lactams in obese critically ill patients. Antimicrob Agents Chemother 2013;57:708-15.

26. ProCESS Investigators, Yealy DM, Kellum JA, et al. A randomized trial of protocol-based care for early septic shock. N Engl J Med 2014;370:1683-93.

27. Weil MH, Shubin H. The "VIP" approach to the bedside management of shock. JAMA 1969;207:337-40.

28. De Backer D, Biston P, Devriendt J, et al. Comparison of dopamine and norepinephrine in the treatment of shock. N Engl J Med 2010;362:779-89.

29. De Backer D, Aldecoa C, Njimi H, Vincent JL. Dopamine versus norepinephrine in the treatment of septic shock: a meta-analysis. Crit Care Med 2012;40:725-30.

30. Jones $A E$, Shapiro NI, Trzeciak $\mathrm{S}$, et al. Lactate clearance vs central venous oxygen saturation as goals of early sepsis therapy: a randomized clinical trial. JAMA 2010;303:739-46.

31. Piagnerelli $M$, Van Nuffelen $M$, Maetens $Y$, Lheureux $P$, Vincent JL. A 'shock room' for early management of the acutely ill. Anaesth Intensive Care 2009;37:426-31.

32. Carlbom DJ, Kupchik N, Calogero V, Switzer D, Utarnachitt R. Code sepsis: improving survival by activation of a critical care team. Am J Respir Crit Care Med 2012;185:A4002.

33. Champunot R, Kamsawang N, Tuandoung P, Tansuphaswasdikul S. Saving 500 Lives Campaign: another way to improve the mortality rate of patients with severe sepsis and septic shock. Crit Care 2012;16(Suppl 3):P105. 